\title{
Power Method on Boundary Value Problems
}

\author{
Madhumita Gogoi Konwar \\ Naharkatia H.S. School \\ Dibrugarh, Assam, India
}

\author{
Arun Kumar Baruah \\ Department of Mathematics \\ Dibrugarh University, Assam, India
}

\begin{abstract}
Matrix equations obtained as a result of separation of parameters of a two-parameter eigenvalue problem in the form of second order ordinary differential equation satisfying certain boundary conditions are considered in the paper. Power method is applied to obtain the greatest and the smallest eigenvalues and their corresponding eigenvectors of the problem. A numerical example is given in support of the method.
\end{abstract}

\section{Keywords}

Matrix, eigenvalue, eigenvector, two-parameter problem, linear ordinary differential equation, boundary value problem.

\section{INTRODUCTION}

The eigenvalues and eigenvectors of a matrix are needed for many applications. One such application arises in solving linear ordinary differential equations. Computation of matrix eigenvalues and eigenvectors is one of the most important problems in numerical analysis. The eigenvalue computation is identical with the problem of computing zero of a polynomial, where as the eigenvector determination reduces the problem of solving a set of homogeneous equations.

In this paper power method is employed to find out the eigenvectors corresponding to the greatest and smallest eigenvalues of a matrix, obtained after transforming an ordinary differential equation into finite difference form.

\section{TWO-PARAMETER PROBLEM}

The aim of this section is to deal with two-parameter problems and discuss its theoretical aspects which are necessary for the present work. The problem considered here is in the form of the three point boundary value problem of the type

$\frac{d^{2} y(x)}{d x^{2}}+\left\{\lambda_{1}+\lambda_{2} f(x)+g(x)\right\} y(x)=0$

associated with boundary conditions

$$
y(a)=y(b)=y(c)=0
$$

where $a<b<c, f(x) \& g(x)$ are given continuous functions of the independent variable $x \in[a, c], f(x)$ is monotonic in $x \in[a, c]$.

In order to progress with the theoretical aspects first, it is necessary to introduce certain definitions pertaining to the above problem.

\subsection{Definition}

If the equation (2.1) subject to the boundary condition (2.2) is satisfied by the pair $\left(\lambda_{1}, \lambda_{2}\right)=\left(\lambda_{1 r}, \lambda_{2 r}\right)$ and $y(x)=y_{r}(x) \neq 0$, then the pair $\left(\lambda_{1 r}, \lambda_{2 r}\right)$ is said to be an eigenvalue pair and $y_{r}(x)$ an eigenfunction corresponding to the eigenvalue pair $\left(\lambda_{1 r}, \lambda_{2 r}\right)$ of the problem (2.1), (2.2).

\subsection{Definition}

The point at which an eigenfunction $y(x)$ of the twoparameter problem (2.1), (2.2) vanishes in the interval $[a, b]$ or $[b, c]$ are called zeros (oscillating points) of the eigenfunction.

\subsection{Definition}

An eigenfunction of the two parameter problem (2.1), (2.2) is said to be oscillatory in respective interval i.e. either in $[a, b]$ or $[b, c]$ if there exist at least one zero in the interval.

From the boundary conditions (2.2) imposed on the problem, one must ensure of eigenfunctions which are at best oscillatory in the present case.

Now the theorem cited in [1] for the real eigenvalue for the parameters $\lambda_{1} \& \lambda_{2}$ is as below :

\subsection{Theorem}

If $a, b, c$ are real with $a<b<c, f(x), g(x)$ are real for $a \leq b \leq c$ and $f(x)$ is monotonic for $a \leq x \leq c$, and the equation has no singularities in $a \leq x \leq c$, then the eigenvalue pair of the problem (2.1), (2.2) are all real.

All the conditions of the theorem are satisfied in the present consideration. Hence, in the latter part of the discussion the eigenvalue pairs of the problem (2.1), (2.2) are considered as real.

The most common approach to the homogeneous twoparameter problem (2.1), (2.2) is to reduce it into another pair of equations each of which contains one parameter only. In this context a discussion on the reformulation of the problem in the Hilbert space under consideration may found in [2].

To reformulate the problem (2.1), (2.2) in the tensor product of Hilbert spaces and separate the parameters involved in the problem, first the problem is written as the pair of equations:,

$$
\frac{d^{2} y_{1}\left(x_{1}\right)}{d x_{1}{ }^{2}}+\left\{\lambda_{1}+\lambda_{2} f_{1}\left(x_{1}\right)+g_{1}\left(x_{1}\right)\right\} y_{1}\left(x_{1}\right)=0
$$

subject to the boundary conditions 


$$
y_{1}\left(a_{1}\right)=y_{1}\left(b_{1}\right)=0
$$

in the Hilbert space $H_{1}=L^{2}\left(\left[a_{1}, b_{1}\right]\right)$, and

$$
\frac{d^{2} y_{2}\left(x_{2}\right)}{d x_{2}{ }^{2}}+\left\{\lambda_{1}+\lambda_{2} f_{2}\left(x_{2}\right)+g_{2}\left(x_{2}\right)\right\} y_{2}\left(x_{2}\right)=0
$$

subject to the boundary conditions

$$
y_{2}\left(a_{2}\right)=y_{2}\left(b_{2}\right)=0
$$

in the Hilbert space $H_{2}=L^{2}\left(\left[a_{2}, b_{2}\right]\right)$,

where $a_{1}=a, b_{1}=b=a_{2}, b_{2}=c$ such that

$f_{1}\left(x_{1}\right)$ in $x_{1} \in\left[a_{1}, b_{1}\right]=f(x)$ in $x \in[a, b]$,

$g_{1}\left(x_{1}\right)$ in $x_{1} \in\left[a_{1}, b_{1}\right]=g(x)$ in $x \in[a, b]$,

$f_{2}\left(x_{2}\right)$ in $x_{2} \in\left[a_{2}, b_{2}\right]=f(x)$ in $x \in[b, c]$ and

$g_{2}\left(x_{2}\right)$ in $x_{2} \in\left[a_{2}, b_{2}\right]=g(x)$ in $x \in[b, c]$.

Combining the above equations, the problem represented in $H=H_{1} \otimes H_{2}$ as

$$
\frac{d^{2} y_{r}\left(x_{r}\right)}{d x_{r}^{2}}+\left\{\lambda_{1}+\lambda_{2} f_{r}\left(x_{r}\right)+g_{r}\left(x_{r}\right)\right\} y_{r}\left(x_{r}\right)=0, r=1,2 .
$$

with boundary conditions

$$
y_{r}\left(a_{r}\right)=y_{r}\left(b_{r}\right)=0, r=1,2 .
$$

Now, applying the definition (2.1), for an eigenvalue pair $\left(\lambda_{1}, \lambda_{2}\right)$ for which both the equations in (2.7), (2.8) possess a common non-trivial solution of (2.7), (2.8) corresponding to a eigenvalue pair $\left(\lambda_{1}, \lambda_{2}\right)$ is also a solution of the original problem (2.1), (2.2). Few relevant definitions in this context are :.

\subsection{Definition}

A pair $\left(\lambda_{1}, \lambda_{2}\right)$ is called an eigenvalue pair of the problem (2.7), (2.8) if the pair of equations represented by the problem possess a common nontrivial solution $y_{r}$ in $H_{r}, r=1,2$. The tensor product $Y_{1} \otimes Y_{2}$ of the solutions is called an eigenvector of the problem corresponding to the eigenlue pair $\left(\lambda_{1}, \lambda_{2}\right)$.

Tensor product of each equation in (2.7), (2.8) by the $y_{r}$ not appearing in that equation are taken. Then the equations of the problem (2.7), (2.8) can be transformed to a pair of simultaneous equations in $H_{1} \otimes H_{2}=L^{2}\left(\left[a_{1}, b_{1}\right] \times\left[a_{2}, b_{2}\right]\right)$ in the form

$$
\frac{d^{2} Y}{d x_{r}{ }^{2}}+\left\{\lambda_{1}+\lambda_{2} f_{r}\left(x_{r}\right)+g_{r}\left(x_{r}\right)\right\} Y=0, r=1,2 .
$$

where $Y\left(x_{1}, x_{2}\right)=y\left(x_{1}\right) \otimes y\left(x_{2}\right)$ and associated with rectangular boundary conditions

$$
\begin{aligned}
& Y\left(a_{1}, x_{2}\right)=Y\left(b_{1}, x_{2}\right)= \\
& Y\left(x_{1}, a_{2}\right)=Y\left(x_{1}, b_{2}\right)=0
\end{aligned}
$$

\subsection{Definition}

A pair $\left(\lambda_{1}, \lambda_{2}\right)$ is called an eigenvalue pair of the problem (2.9), (2.10) if the equations possess a common non-trivial solution $\quad Y$ in $\bigcap_{r=1}^{2} D\left(\frac{\partial^{2}}{\partial x_{r}^{2}}+g_{r}\right)$. The common non-trivial solution $Y$ is called an eigenvector of the problem (2.9), (2.10) corresponding to the eigenvalue pair $\left(\lambda_{1}, \lambda_{2}\right)$.

The problem (2.9), (2.10) is meaningful for not only decomposable vectors $Y$, but also for some other vectors in $H$. In that case, the problem will represent a pair of simultaneous equations both posed in the same space $H$. The operators in one equation commute with the operators in the other equation. However, the two problems in (2.7), (2.8) and (2.9), (2.10) are equivalent as eigenvalue problems (Baruah [2]).

On separating the pair of parameters $\lambda_{1}$ and $\lambda_{2}$ from the differential equation (2.9), the reduced form of the problem (2.9), (2.10) obtained as the pair of partial differential equations,

$$
\frac{\left[-f_{2}\left(x_{2}\right) \frac{\partial^{2}}{\partial x^{2}}+f_{1}\left(x_{1}\right) \frac{\partial^{2}}{\partial x^{2}}-f_{2}\left(x_{2}\right) g_{1}\left(x_{1}\right)+f_{1}\left(x_{1}\right) g_{2}\left(x_{2}\right)\right]_{Y}}{f_{2}\left(x_{2}\right)-f_{1}\left(x_{1}\right)}=\lambda_{1} Y
$$

and $\frac{\left[\frac{\partial^{2}}{\partial x_{1}^{2}}-\frac{\partial^{2}}{\partial x_{2}^{2}}+g_{1}\left(x_{1}\right)-g_{2}\left(x_{2}\right)\right] Y}{f_{2}\left(x_{2}\right)-f_{1}\left(x_{1}\right)}=\lambda_{2} Y$

where $Y\left(x_{1}, x_{2}\right)=y\left(x_{1}\right) \otimes y\left(x_{2}\right)$, each of which is subject to the rectangular boundary conditions (2.10) assuming, $f_{2}\left(x_{2}\right)-f_{1}\left(x_{1}\right) \neq 0$,

\subsection{Definition}

A pair $\left(\lambda_{1}, \lambda_{2}\right)$ is called an eigenvalue pair of the reduced problem (2.11), (2.12), (2.10) if the equations possess a common non-trivial solution $Y$ in

$$
\begin{array}{r}
D\left(\frac{-f_{2} \frac{\partial^{2}}{\partial x^{2}}+f_{1} \frac{\partial^{2}}{\partial x^{2}}-f_{2} g_{1}+f_{1} g_{2}}{f_{2}-f_{1}}\right) \\
\cap D\left(\frac{\frac{\partial^{2}}{\partial x_{1}^{2}}-\frac{\partial^{2}}{\partial x_{2}^{2}}+g_{1}-g_{2}}{f_{2}-f_{1}}\right)
\end{array}
$$

and the common solution is called an eigenvector of the problem (2.11), (2.12), (2.10) corresponding to the eigenvalue pair $\left(\lambda_{1}, \lambda_{2}\right)$.

Clearly, an eigenvalue pair of the problem (2.7), (2.8) with an eigenvector $Y=Y_{1} \otimes Y_{2}$ is also an eigenvalue pair of the reduced problem (2.11), (2.12), (2.10) with the same eigenvector $Y$. But the converse is not necessarily true, because an eigenvector of the problem (2.11), (2.12), (2.10) may not be decomposable at all. But in the present case, the two problems are equivalent problems. Hence corresponding to an eigenvalue pair $\left(\lambda_{1}, \lambda_{2}\right)$, the eigenvector $Y$ of $(2.11),(2.12),(2.10)$ is then decomposable in to $Y=Y_{1} \otimes Y_{2}$, where $Y_{1}$ and $Y_{2}$ are common non-trivial solutions of the problem (2.7), 
(2.8). The two solutions $Y_{1}$ and $Y_{2}$ are thus two eigenvectors of the original problem (2.1), (2.2) corresponding to the eigenvalue pair $\left(\lambda_{1}, \lambda_{2}\right)$.

Thus, the original problem (2.1), (2.2) written in the form (2.7), (2.8) has been reduced to a pair of one parameter problems $(2.11),(2.12),(2.10)$ in the tensor product $H=H_{1} \otimes H_{2}$. For the present case of the two-parameter problem in the form of ordinary differential equations (2.1), (2.2) can be interpreted as the replacement of the problem equivalently by a pair of one- parameter partial differential equations (2.11), (2.12) each of which is subject to the rectangular boundary conditions (2.10).

Next, using the equal interval size ' $h$ ' and replacing the derivatives in $(2.11),(2.12)$ by their corresponding central difference approximations, the difference equations obtained as below :

$\left[h^{2}\left\{\lambda_{1}\left(f_{2}\left(x_{2}\right)-f_{1}\left(x_{1}\right)\right)-f_{1}\left(x_{1}\right) g_{2}\left(x_{2}\right)+\right.\right.$ $\left.\left.f_{2}\left(x_{2}\right) g_{1}\left(x_{1}\right)\right\}+2 f_{1}\left(x_{1}\right)-2 f_{2}\left(x_{2}\right)\right] y\left(x_{1}, x_{2}\right)+$ $f_{2}\left(x_{2}\right)\left\{y\left(x_{1}+h, x_{2}\right)+y\left(x_{1}-h, x_{2}\right)\right\}-f_{1}\left(x_{1}\right)\left\{y\left(x_{1}, x_{2}+\right.\right.$ h) $\left.+y\left(x_{1}, x_{2}-h\right)\right\}=0$

(2.13) and

$\left[h^{2}\left\{\lambda_{2}\left(f_{2}\left(x_{2}\right)-f_{1}\left(x_{1}\right)\right)-\left(g_{1}\left(x_{1}\right)-g_{2}\left(x_{2}\right)\right)\right\}\right] y\left(x_{1}, x_{2}\right)-$ $\left\{y\left(x_{1}+h, x_{2}\right)+y\left(x_{1}-h, x_{2}\right)\right\}+\left\{y\left(x_{1}, x_{2}+h\right) y\left(x_{1}, x_{2}-\right.\right.$ h) $\}=$

From these two finite difference representations (2.13), (2.14) two linear system of equations can be obtained and from which one can find the coefficient matrices. To these coefficient matrices Power Method can be applied to find out solutions which will give a solution for the boundary value problem. A numerical example is given in the next section.

\section{NUMERICAL EXAMPLE}

The boundary value problem considered is

$$
y^{\prime \prime}+\left(\lambda+\mu \cos x+e^{x}\right) y=0,
$$

associated with the boundary conditions

$$
y(0)=y(2)=y(4)=0
$$

Applying the separation of parameters to this problem two single parameter problems are obtained. Then representing them in finite difference form as discussed in the previous section the equations obtained are :

$\left[h^{2}\left\{\lambda\left(\cos x_{2}-\cos x_{1}\right)-\cos x_{1} e^{x_{2}}+\cos x_{2} e^{x_{1}}\right\}+\right.$ $\left.2 \cos x_{1}-2 \cos x_{2}\right] y\left(x_{1}, x_{2}\right)+\cos x_{2}\left\{y\left(x_{1}+h, x_{2}\right)+\right.$ $\left.y\left(x_{1}-h, x_{2}\right)\right\}-\cos x_{1}\left\{y\left(x_{1}, x_{2}+h\right)+y\left(x_{1}, x_{2}-h\right)\right\}=0$

And $\left[h^{2}\left\{\mu\left(\cos x_{2}-\cos x_{1}\right)-\left(e^{x_{1}}-e^{x_{2}}\right)\right\}\right] y\left(x_{1}, x_{2}\right)-$ $\left\{y\left(x_{1}+h, x_{2}\right)+y\left(x_{1}-h, x_{2}\right)\right\}+\left\{y\left(x_{1}, x_{2}+h\right) y\left(x_{1}, x_{2}-\right.\right.$ h) $\}=$

subject to

$$
y\left(0, x_{2}\right)=y\left(2, x_{2}\right)=y\left(x_{1}, 2\right)=y\left(x_{1}, 4\right)=0
$$

Now the value of the functions $y\left(x_{1}, x_{2}\right)$ with $h=1 / 2$, the lattices points of the rectangular boundary are given by

$$
\begin{gathered}
y_{1}=\left(\frac{1}{2}, \frac{7}{2}\right), y_{2}=\left(1, \frac{7}{2}\right), y_{3}=\left(\frac{3}{2}, \frac{7}{2}\right), \\
y_{4}=\left(\frac{1}{2}, 3\right), y_{5}=(1,3), y_{6}=\left(\frac{3}{2}, 3\right) \\
y_{7}=\left(\frac{1}{2}, \frac{5}{2}\right), y_{8}=\left(1, \frac{5}{2}\right), y_{9}=\left(\frac{3}{2}, \frac{5}{2}\right), \\
\quad(3.6)
\end{gathered}
$$

The difference equations (3.3) at these points are as below :

$$
\begin{gathered}
\alpha_{1} y_{1}+\beta_{1} y_{2}-\gamma_{1} y_{4}=0, \\
\alpha_{1}=-.00045 \lambda-7.8634, \\
\beta_{1}=.9981, \gamma_{1}=.9999 \\
\alpha_{2} y_{2}+\beta_{2} y_{3}+\beta_{2} y_{1}-\gamma_{2} y_{5}=0, \\
\alpha_{2}=-.00043 \lambda-7.5959, \\
\beta_{2}=.9981, \gamma_{2}=.9998 \\
\alpha_{3} y_{3}+\beta_{3} y_{2}-\gamma_{3} y_{6}=0, \\
\alpha_{3}=-.00037 \lambda-7.1545, \\
\beta_{3}=.9981, \gamma_{3}=.9997 \\
\alpha_{4} y_{4}+\beta_{4} y_{5}-\gamma_{4} y_{1}-\gamma_{4} y_{7}=0, \\
\alpha_{4}=-.00033 \lambda-4.6096, \\
\beta_{4}=.9986, \gamma_{4}=.9999 \\
\alpha_{5} y_{5}+\beta_{5} y_{6}+\beta_{5} y_{4}-\gamma_{5} y_{2}-\gamma_{5} y_{8}=0, \\
\alpha_{5}=-.00030 \lambda-4.3394, \\
\beta_{5}=.9986, \gamma_{5}=.9998 \\
\alpha_{6} y_{6}+\beta_{6} y_{5}-\gamma_{6} y_{3}-\gamma_{6} y_{9}=0, \\
\alpha_{6}=-.00026 \lambda-3.8987, \\
\beta_{6}=.9986, \gamma_{6}=.9997 \\
\alpha_{8} y_{8}+\beta_{8} y_{9}+\beta_{8} y_{7}-\gamma_{8} y_{5}=0, \beta_{9} y_{8}-\gamma_{9} y_{6}=0, \\
\alpha_{9}=-.00015 \lambda-1.9240, \\
\beta_{7}=.9990, \gamma_{7}=.9999 \\
\alpha_{7} y_{7}+\beta_{7} y_{8}-\gamma_{7} y_{4}=0, \\
\alpha_{7}=-.00023 \lambda-2.6319,
\end{gathered}
$$$$
\beta_{9}=.9990, \gamma_{9}=.9997
$$ 
Putting $\lambda=-4.6204$ taken from [3] in the above system of equations will give the coefficient matrix A as below :

$$
A=\left[\begin{array}{ccccccccc}
-7.8613 & 0.9981 & 0 & -0.9999 & 0 & 0 & 0 & 0 & 0 \\
0.9981 & -7.5939 & 0.9981 & 0 & -0.9998 & 0 & 0 & 0 & 0 \\
0 & 0.9981 & -7.1529 & 0 & 0 & -0.9997 & 0 & 0 & 0 \\
-0.9999 & 0 & 0 & -4.6081 & .9986 & 0 & -0.9999 & 0 & 0 \\
0 & -0.9998 & 0 & 0.9986 & -4.3381 & 0.9986 & 0 & -0.9998 & 0 \\
0 & 0 & -0.9997 & 0 & 0.9986 & -3.8974 & 0 & 0 & -0.9997 \\
0 & 0 & 0 & -0.9999 & 0 & 0 & -2.6308 & 0.999 & 0 \\
0 & 0 & 0 & 0 & -0.9998 & 0 & 0.999 & -2.3637 & 0.999 \\
0 & 0 & 0 & 0 & 0 & -0.9997 & 0 & 0.999 & -1.9233
\end{array}\right]
$$

Now, applying the power method ( [4], [5] ) to the matrix ' $A$ ' after 52 iterations (using Mathemetica) the greatest eigenvalue of the matrix ' $A$ ' is found to be '$9.30045^{\prime}$ and the corresponding eigenvector as,

$$
\left[\begin{array}{lllllllll}
-0.87360 & 1 & -0.53917 & -0.25912 & 0.29661 & -0.15993 & -0.04688 & 0.05367 & -0.02894
\end{array}\right]^{T}
$$

This is also the eigenvector corresponding to $\lambda=$ -4.6204 .

Similarly for the difference equations (3.4) for $\mu=7.8787$ taken from
[2], the greatest eigenvalue of the coefficient matrix $\mathrm{B}$ is found to be '9.3051' and its corresponding eigenvector as,

$$
\left[\begin{array}{lllllllll}
-.87323 & 1 & -0.53951 & -0.25896 & 0.29655 & -0.15999 & -0.04684 & 0.05364 & -0.02894
\end{array}\right]^{T}
$$

Thus, it is seen that the eigenvectors (3.9), (3.10) are almost equal and hence one can say that
$(-4.6204,7.8787)$ is an eigenvalue pair with the corresponding eigenvector,

$$
\left[\begin{array}{lllllllll}
-.8732 & 1 & -0.5395 & -0.2589 & 0.2965 & -0.1599 & -0.0468 & 0.0536 & -0.0289
\end{array}\right]^{T}
$$

In the same process, it is easy to verify that $(-4.32$, $24.37),(9.11,23.10)$ are also eigenvalue pairs. And for these eigenvalue pairs it was found that greatest

eigenvalue pairs of the matrices $\mathrm{A}$ and $\mathrm{B}$ as $(-9.3007$, 9.2984), $(-9.3048,9.2990) \quad \&$ their corresponding eigenvectors are (approximately)

$$
\begin{aligned}
& {\left[\begin{array}{lllllllll}
-0.873 & 1 & -0.539 & -0.259 & 0.297 & -0.160 & -0.047 & 0.054 & 0.029
\end{array}\right]^{T} \&} \\
& {\left[\begin{array}{lllllllll}
-0.87 & 1 & -0.54 & -0.26 & 0.297 & -0.160 & -0.047 & 0.054 & 0.029
\end{array}\right]^{T}}
\end{aligned}
$$

After obtaining the dominant eigenvalues of the two matrices ' $A$ ' and ' $B$ ' shifting of origin ([6], [7]) are applied to these matrices to obtain the smallest eigenvalues as ' -0.415 ' and ' 0.414 ' respectively and their corresponding eigenvector is

$$
\left[\begin{array}{lllllllll}
0.044 & 0.078 & 0.074 & -0.251 & -0.445 & -0.419 & -0.565 & 1 & 0.939
\end{array}\right]^{T} \text {. }
$$

\section{CONCLUSION}

In this paper Power method is illustrated with the help of the numerical example (3.1), (3.2) after separation of parameters $\lambda$ and $\mu$. Here it is verified that ($4.6204,7.8787),(-4.32,24.37),(9.11,23.10)$ are eigenvalue pairs. While it was attempted to verify the eigenvalue pair $(-4.6204,7.8787)$, it was found that the matrices ' $A$ ' and ' $B$ ' of which the greatest eigenvalue pair is $(-9.30045,9.30512)$ and their respective eigenvectors are also found, which are equivalent to the eigenvectors of $(-4.6204,7.8787)$ and thus gives a solution to the boundary value problem (3.1),(3.2). After that by using the concept of shifting of origin, the smallest eigenvalue pair $(-0.415,0.414)$ and its corresponding eigenvector are also obtained. 


\section{REFERENCES}

[1] Arscott, F. M., 1968, Two-parameter eigenvalue problems in the differential equations, Proc. London Math. Soc.(3) 14, pp. (459 - 470).

[2] Baruah, A. K., 1987, Estimation of eigenelements in a two-parameter eigenvalue problem, Ph.D. Thesis, Dibrugarh University, Assam.

[3] Fox, L. Hayes, L \& Mayers, D. F., 1972, The double eigenvalue problem : Topics in numerical analysis, Proc. Roy. Irish Acad. Con., Univ. College, Dublin, Academic Press, pp. (93-112).
[4] Gerald, Curtis F. \& Wheatley Patrick O., 1994, Applied numerical analysis, Addison-Wesley Longman Publishing Company, California.

[5] Burden, R. L. \& Faires, J. D., 1997, Numerical Analysis, Sixth Edition, Brooks/ Cole Publishing Company, New York.

[6] Jain, M. K., Iyengar, S. R. K. and Jain, R. K., 1997, Computational methods for partial differential equations, New Age International Publishers, New Delhi.

[7] Jain, M. K., Iyengar, S. R. K. and Jain, R. K., 2007, Numerical method for scientific and engineering computation, $5^{\text {th }}$ Edition, New Age_International Publishers, New Delhi. 